\title{
Recent Advances in Molecular Scale Electronics
}

\author{
JAMES M. TOUR,${ }^{a, b}$ WILLIAM A. REINERTH,${ }^{b}$ LEROY JONES II, ${ }^{b}$ \\ TIMOTHY P. BURGIN, ${ }^{b}$ CHONG-WU ZHOU, ${ }^{c}$ C. J. MULLER, ${ }^{c}$ M.R. DESHPANDE, ${ }^{c}$ \\ AND MARK A. REED ${ }^{c}$ \\ ${ }^{b}$ Department of Chemistry and Biochemistry, University of South Carolina, 730 South \\ Main Street, Columbia, South Carolina 29208, USA \\ ${ }^{c}$ Department of Electrical Engineering, Yale University, New Haven, Connecticut 06520, \\ $U S A$
}

ABSTRACT: This paper describes four significant breakthroughs in the syntheses and testing of molecular scale electronic devices. The 16-mer of oligo(2-dodecylphenylene ethynylene) was prepared on Merrifield's resin using the iterative divergent/convergent approach which significantly streamlines the preparation of this molecular scale wire. The formation of self-assembled monolayers (SAMs) and multilayers on gold surfaces of rigid rod conjugated oligomers that have thiol, $\alpha, \omega$-dithiol, thioacetyl, or $\alpha, \omega$-dithioacetyl end groups have been studied. The direct observation of charge transport through molecules of benzene-1,4-dithiol, which have been self-assembled onto two facing gold electrodes, has been achieved. Finally, we are reporting initial studies into what effect varying the molecular alligator clip has on the molecular scale wire's conductivity.

\section{INTRODUCTION}

Future computational systems will likely consist of logic devices that are ultra dense, ultra fast, and molecule-sized. ${ }^{1-23}$ The slow step in existing computational architectures is not usually the switching time, but the time it takes for an electron to travel between devices. By using molecular scale electronic interconnects, ${ }^{d, 24}$ the transmit times could be minimized, resulting in computational systems that operate at far greater speeds than is presently attainable from conventional patterned architectural arrays. ${ }^{1}$ There is another technical advantage that might also be gained from molecular scale devices. A powerful computational system presently utilizes about $10^{10}$ silicon-based devices. If devices were to be based upon single molecules, ${ }^{21}$ using routine chemical syntheses, one could prepare approximately $10^{23}$ devices in a single reaction flask. Of course, the task of addressing large arrays of ordered molecular scale devices is presently unattainable; however, the potential is certainly enough to maintain current and future interests.

${ }^{a}$ Address for telecommunication: Phone, 803/777-9517; fax, 803/777-9521.

$d$ "Molecular electronics" is a poorly defined term since some authors refer to it as any molecular-based system, such as a film or a liquid crystalline array. Other authors, including us, have preferred to reserve the term "molecular electronics" for single-molecule tasks, such as single molecule-based devices or single molecular wires. Because of this confusion, we have chosen here to follow the Petty et al. terminology by using two subcategories, namely "molecular materials for electronics" for bulk applications and "molecular scale electronics" for single molecule applications. 
Though it is well-documented that bulk conjugated organic materials can be semiconducting or even conducting when doped, ${ }^{25}$ we only recently determined how thiol-ended rigid rod conjugated molecules orient themselves on gold surfaces, ${ }^{26,27}$ and how we could record electronic conduction through single undoped conjugated molecules that are end-bound onto a metal probe surface. ${ }^{28}$ We have previously described the syntheses of soluble oligo(3-ethyl-2,5-thiophene-ethynylene)s and oligo(2-alkyl-1,4-phenylene-ethynylene)s, potential molecular scale wires, by a rapid, solution phase iterative divergent/convergent doubling approach, ${ }^{29,32}$ as well as the syntheses and attachments of protected thiol moieties to one or both ends of the molecular scale wires. These thiols serve as molecular scale alligator clips for adhesion of the molecular scale wires to the gold probes. ${ }^{26-28}$

Herein we report four significant breakthroughs that could have dramatic implications in the syntheses and testing of molecular scale electronic devices. First, we have completed the synthesis of the dodecyl-containing 16-mer on Merrifield's resin (chloromethyl polystyrene) using the iterative divergent/convergent approach, a method which significantly streamlines the preparation. ${ }^{33-36}$ Secondly, we have studied the formation of self-assembled monolayers (SAMs) and multilayers on gold surfaces of rigid rod conjugated oligomers that have thiol, $\alpha, \omega$-dithiol, thioacetyl, or $\alpha, \omega$-dithioacetyl end groups. Thirdly, molecules of benzene-1,4-dithiol have been self-assembled onto the two facing gold electrodes of a mechanically controllable break junction (MCB) to form a statically stable gold-sulfur-aryl-sulfur-gold system, which allows for direct observation of charge transport through the molecules. Finally, we are examining what effect varying the molecular alligator clip has on the molecular scale wire's conductivity.

\section{RESULTS AND DISCUSSION}

\section{Solid Phase Oligomer Synthesis}

The monomers needed for the polymer supported synthesis were prepared as depicted in SCHEME I. The silylated triazene was divided into two portions; the first portion was desilylated to form $\mathbf{1}$, the anchor unit to be attached to the polymer support, while the second portion was iodinated ${ }^{33-36}$ to form the iodoarene 2. Attachment of 1 to the polymer support resin, and the oligomer syntheses on the polymer support are depicted in SCHEME II. This polymer support approach greatly simplifies the isolation and purification of oligomers $5,8,11$, and 14 .

\section{Self-Assembled Monolayer Formation of Rigid-Rod Conjugated Oligomers}

As a prelude to the utilization of molecular scale wires in molecular scale electronic devices, it is necessary to understand the molecular ordering on metal surfaces. These sulfur-terminated conjugated oligomers form self-assembled monolayers (SAMs) on gold surfaces by attachment of the thiol end groups which serve as molecular scale alligator clips. ${ }^{26}$ The SAMs were analyzed using ellipsometry, X-ray photoelectron spectroscopy (XPS), and infrared external reflectance spectroscopy. The thiol moieties usually dominate adsorption on the gold sites; interactions with the conjugated $\pi$-systems are weaker. Rigid rod $\alpha, \omega$-dithiols form assemblies in which one thiol group binds to the surface while the second thiol moiety projects up- 
ward at the exposed surface of the SAM. In situ deprotection of the thiol moieties by deacylation of thioacetyl groups using $\mathrm{NH}_{4} \mathrm{OH}$ permits formation of SAMs without having to isolate the oxidatively unstable free thiols. Moreover, direct adsorption, without exogenous base, of the thioacetyl-terminated oligomers can be accomplished to generate gold surface-bound thiolates. However, in the non-base-promoted adsorptions, higher concentrations of the thioacetyl groups, relative to that of thiol groups, are required to achieve monolayer coverage in a given interval. A thiolterminated phenylene ethynylene system was shown to have a tilt angle of the long molecular axis of $<20^{\circ}$ from the normal to the substrate surface. These aromatic $\alpha, \omega$-dithiol-derived monolayers provide the basis for studies leading to the design of molecular wires capable of bridging proximal gold surfaces.

\section{SCHEME I}
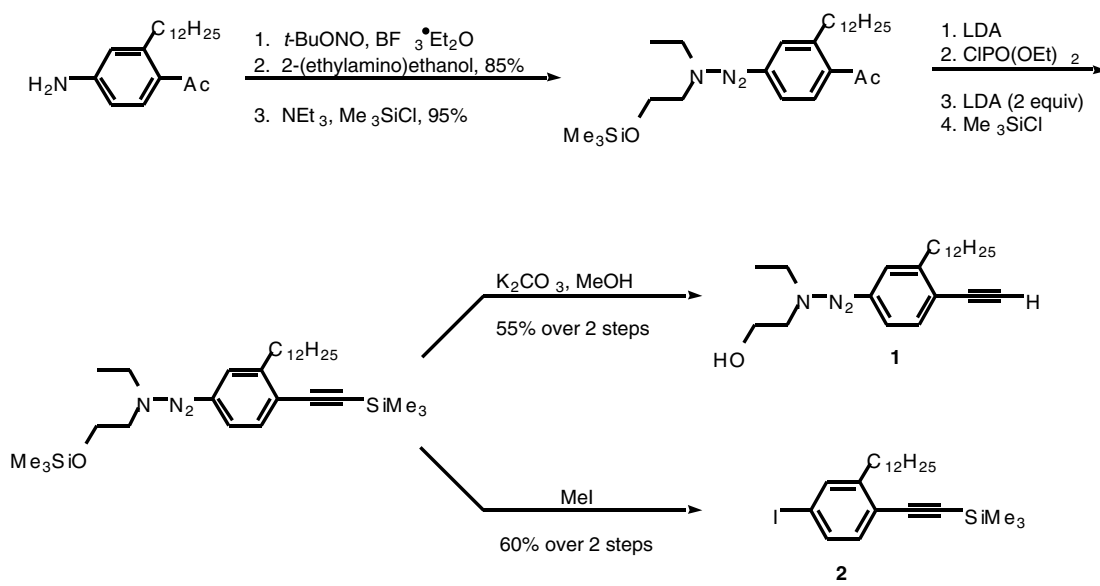

Conductance of a Molecular Junction

Charge transport in and the measurement of the conductance of single organic molecules is an intriguing, experimentally challenging, and long sought goal. These measurements have been performed on benzene-1,4-dithiolate connected between stable proximal metallic gold contacts using a mechanically controllable break junction (MCB). ${ }^{37}$ The metal-molecule-metal configuration presents the molecular embodiment of a system analogous to a quantum dot, with the potential barriers replaced by the contact barrier of the gold-thiolate end groups (FIG. 1). The results show a highly reproducible apparent Coulomb gap at about $0.7 \mathrm{~V}$ at room temperature. This study provides a direct, quantitative measurement of the molecular conductance of a junction containing a single molecule, a fundamental step in the emerging field of molecular scale electronics.

Variation of a Molecular Alligator Clip

By measuring the conductivity of a series of molecules that are systematically altered, the contribution of each component of a molecular scale electronic device can 


\section{SCHEME II}

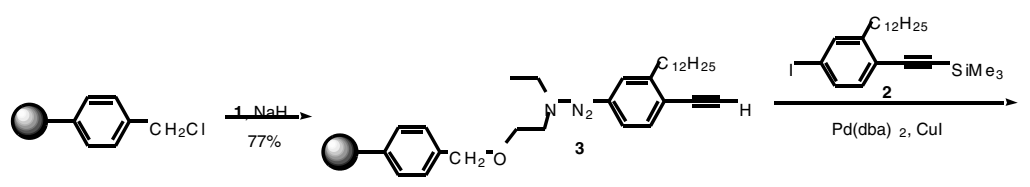

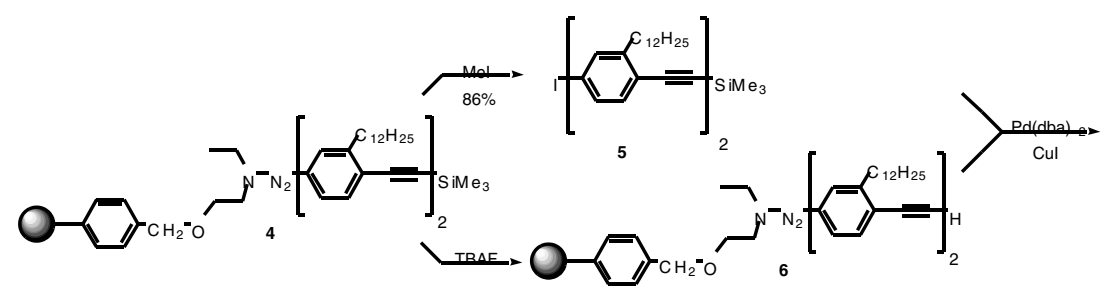

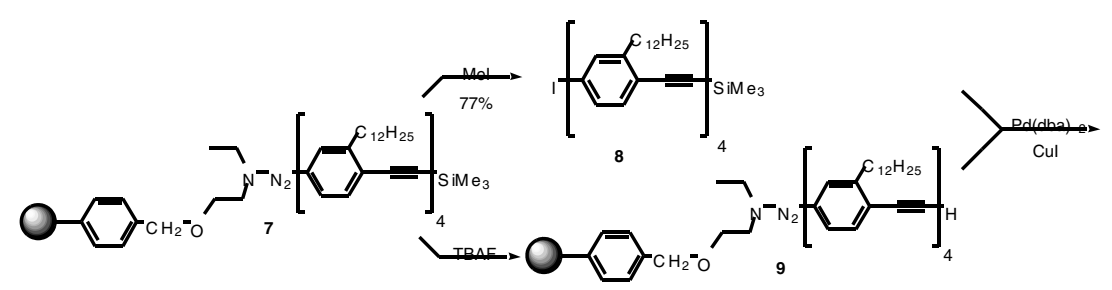

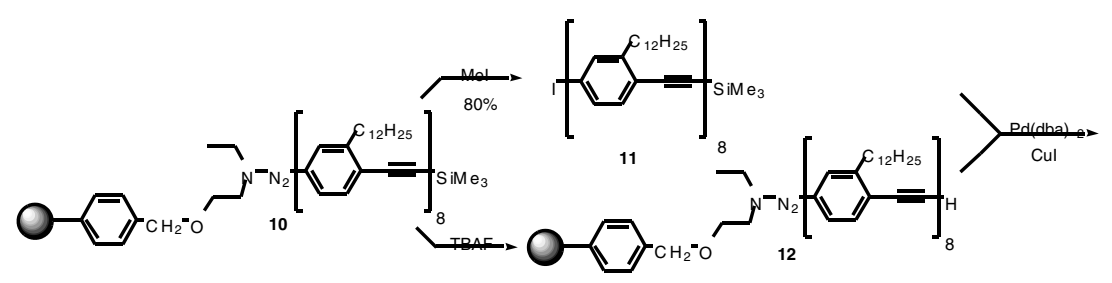<smiles>CCc1cc(N(CC)CCOc2ccc(O)cc2)ccc1C=C(C)C(C)(C)C</smiles>

be determined. For example, the simplest experiment of this type would be to measure the conductivity of identical arrays of molecular wires in which only the wires' attachment to the gold surface is altered. Thus, by varying the molecular alligator clip and examining the molecules' conductivity, the contribution of the alligator clip to the resistance of the molecular wire can be determined. Our initial work has focused on varying the alligator clip by proceeding down the chalcogens from $\mathrm{S}$ to $\mathrm{Se}$ to Te. 


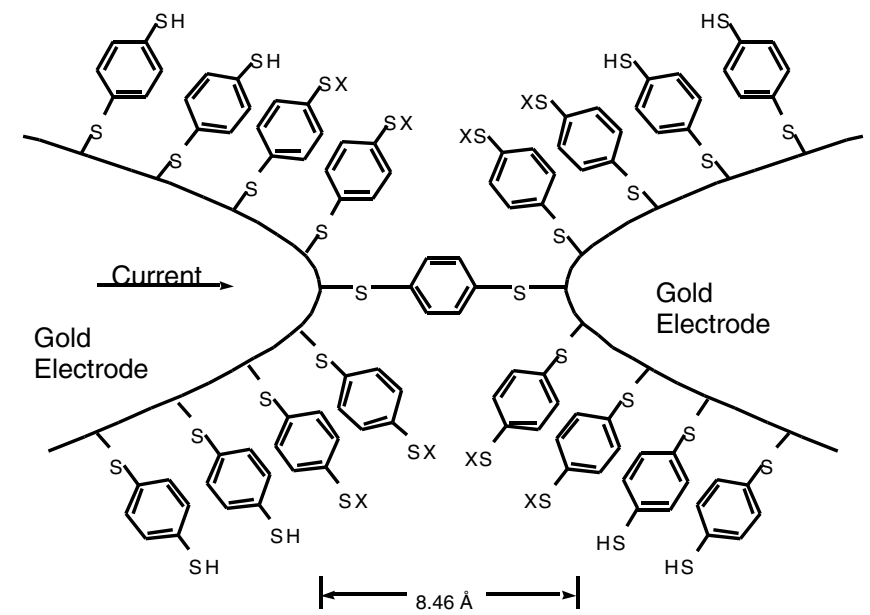

FIGURE 1. The desired placement of benzene-1,4-dithiolate between gold electrodes in a $\mathrm{MCB} .(\mathrm{X}=\mathrm{H}, \mathrm{Au})$.

We first desired a stable, yet easily removable, protecting group for selenium that would allow us to use the same in situ deprotection prototcol we had employed for the thioacetates. An investigation of several protecting groups led us to conclude that, as with sulfur, the acetyl group was the protecting group of choice. ${ }^{38}$ Recently, molecular arrays of 4,4'-biphenyl thioacetate (15), 4,4'-biphenyl selenoacetate (16), and bis $\left(4,4^{\prime}\right.$-biphenyl)ditelluride (17) have been subjected to conductivity examination using a heterostructure nanopore system (FIG. 2).

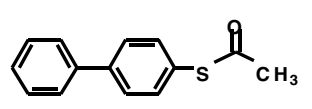

15

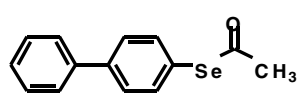

16

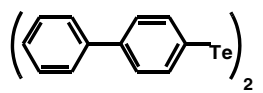

17

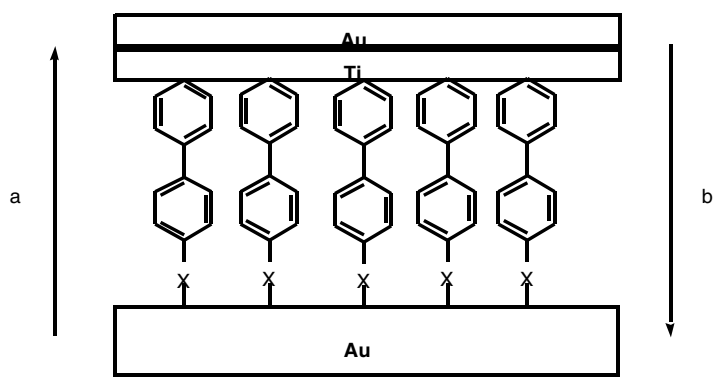

FIGURE 2. Schematic of nanopore experiment $(X=S, S e, T e)$. Arrows indicate direction of electron flow for (a) reverse bias and (b) forward bias. 
We have previously examined the electronic properties of $\mathbf{1 5}$ using this heterostructure nanopore system ( $\mathrm{X}=\mathrm{S}$ in FIG. 2$).{ }^{39}$ Desiring to examine the degree to which the molecule's attachment to the gold surface determines conductivity, we subjected $16(X=S e)$ and $17(X=T e)$ to examination. The barrier heights that were obtained for each of the chalcogen-terminated molecular wires are listed in TABLE 1.

It appears that the selenium alligator clip creates a higher barrier for electron transport regardless of the direction of electron flow. ${ }^{40}$ This is puzzling in light of the greater metallic character of Se versus $S$ which we reasonably assumed would yield a lower barrier for Se. However, tellurium does not exhibit the same trend for barrier height. The Te-terminated molecular wire displays a barrier to conduction comparable to its sulfur congener. This non-periodic trend is puzzling and we are not yet able to fully explain it. However, recent calculations on the HOMO-LUMO gap of chalcogen-terminated aromatics bonded to Au shows a similar trend when the chalcogen bridges three $\mathrm{Au}$ atoms on the surface. ${ }^{41}$

TABLE 1. Barrier heights for chalcogen-terminated biphenyl SAMs

\begin{tabular}{ccc}
\hline $\mathrm{X}$ & Forward bias $(\mathrm{eV})$ & Reverse bias $(\mathrm{eV})$ \\
\hline $\mathrm{S}$ & 0.256 & 0.19 \\
$\mathrm{Se}$ & 0.33 & 0.25 \\
$\mathrm{Te}$ & 0.215 & 0.21 \\
\hline
\end{tabular}

\section{SUMMARY}

In summary, we have made four significant breakthroughs in the syntheses and testing of molecular scale electronic devices. First, we have completed the synthesis of the dodecyl-containing 16-mer on Merrifield's resin (chloromethyl polystyrene) using the iterative divergent/convergent approach; a method which significantly streamlines the preparation. Secondly, we have studied the formation of self-assembled monolayers (SAMs) and multilayers on gold surfaces of rigid rod conjugated oligomers that have thiol, $\alpha, \omega$-dithiol, thioacetyl, or $\alpha, \omega$-dithioacetyl end groups. Thirdly, molecules of benzene-1,4-dithiol have been self-assembled onto the two facing gold electrodes of a mechanically controllable break junction (MCB) to form a statically stable gold-sulfur-aryl-sulfur-gold system, which allows for direct observation of charge transport through the molecules. Finally, we have begun investigating what effect varying the molecular alligator clip has on the molecular scale wire's conductivity. ${ }^{16}$

\section{ACKNOWLEDGMENT}

We thank the Defense Advanced Research Projects Agency for support of this work. 


\section{REFERENCES}

1. Aviram, A., Ed. 1992. Molecular Electronics: Science and Technology. In Conference Proceedings No. 262. American Institute of Physics. New York, NY.

2. BIRGE, R.R., Ed. 1991. Molecular and Biomolecular Electronics. In Advances in Chemistry Series 240. American Chemical Society.

3. KIRK, W.P. \& M.A. ReED, Eds. 1992. Nanostructures and Mesoscopic Systems. Academic Press. New York, NY. In press.

4. MuJiCA, V. et al. 1996. Current-voltage characteristics of molecular wires: Eigenvalue staircase, Coulomb blockade, and rectification. J. Phys. Chem. 104: 72967305.

5. JOACHIM, C. \& J.F. VinUESA. 1996. Length dependence of the electronic transparence (conductance) of a molecular wire. Europhys. Lett. 33: 635-640.

6. Grosshenny, V., A. Harriman \& R. Ziessel. 1995. Electronic energy transfer across ethynyl-bridged $\mathrm{ru}^{\mathrm{ii}} / \mathrm{os}^{\mathrm{ii}}$ terpyridyl complexes. Angew. Chem. Int. Ed. Engl. 34: $1100-1102$.

7. LANGLER, L. et al. 1995. Electrical measurements on submicronic synthetic conductors: carbon nanotubes. Synth. Methods. 70: 1393-1396.

8. PASCUAL, J.I. et al. 1995. Properties of metallic nanowires: from conductance quantization to localization. Science 267: 1793-1795.

9. PuRCELL, S.T. et al. 1994. Characterization of the conductivity of organic thiols by field emission microscopy and field emission spectroscopy. J. Am. Chem. Soc. 116: $11985-11989$.

10. MARTIN, C.R. 1994. Nanomaterials: A membrane-based synthetic approach. Science 266: $1961-1966$.

11. SETH, J. et al. 1994. Investigation of electronic communication in multi-porphyrin light-harvesting arrays. J. Am. Chem. Soc. 116: 10578-10592.

12. GUST, D. 1994. Molecular wires and girders. Nature 372: 133-134.

13. WU, C. \& T. BEIN. 1994. Conducting carbon wires in ordered, nanometer-sized channels. Science 266: 1013-1015.

14. Wagner, R.W. et al. 1996. Molecular optoelectronic gates. J. Am. Chem. Soc. 118: 3996-3997.

15. BRIGELlETti, F. et al. 1994. Rigid rod-like dinuclear ru(ii)/os(ii) terpyridine-type complexes. Electrochemical behavior, absorption spectra, luminescence properties, and electronic energy transfer through phenylene bridges. J. Am. Chem. Soc. 116: 7692-7699.

16. WU, C. \& T. BEIN. 1994. Conducting polyaniline filaments in a mesoporous channel host. Science 264: 1757-1759.

17. MoERnER, W.E. 1994. Examining nanoenvironments in solids on the scale of a single, isolated impurity molecule. Science 265: 46-53.

18. SeSSler J.L., V.L. CAPUANO \& A. HARRIMAN. 1993. Electronic energy migration and trapping in quinone-substituted, phenyl-linked dimeric and trimeric porphyrins. J. Am. Chem. Soc. 115: 4618-4628.

19. FARAZDEL, A. et al. 1990. Electric field induced intramolecular electron transfer in spiro $\pi$-electron systems and their suitability as molecular electronic devices. A theoretical study. J. Am. Chem. Soc. 112: 4206-4214.

20. Tour, J.M., R. WU \& J.S. SCHUMm. 1991. Extended orthogonally fused conducting oligomers for molecular electronic devices. J. Am. Chem. Soc. 113: 7064-7066.

21. DAI, H., E.W. Wong \& C.M. LIEBER. 1996. Probing electrical transport in nanomaterials: Conductivity of individual carbon nanotubes. Science 272: 523-526.

22. WU, R. et al. 1996. Convergent synthetic routes to orthogonally fused conjugated oligomers directed toward molecular scale electronic device applications. J. Org. Chem. 61: 6906-6921.

23. WARD, M.D. 1996. Current developments in molecular wires. Chem. Ind. 568-573. 
24. Petty, M.C. et al., Eds. 1995. Introduction to Molecular Electronics. Oxford University Press. New York, NY. In press.

25. Sкотнеiм, T.A., Ed. 1986. Handbook of Conducting Polymers. Marcel Dekker. New York, NY.

26. Tour, J.M. et al. 1995. Self-assembled monolayers and multilayers of conjugated thiols, $\alpha, \omega$-dithiols, and thioacetyl-containing adsorbates. Understanding attachments between potential molecular wires and gold surfaces. J. Am. Chem. Soc. 117: 9529-9534.

27. DhiRAnI, A. et al. 1996. Self-assembly of conjugated molecular rods: A high resolution STM study. J. Am. Chem. Soc. 118: 3319-3320.

28. BUMM, L.A. et al. 1996. Are single molecular wires conducting? Science 271: 17051707.

29. Schumm, J.S., D.L. PeArson \& J.M. TouR. 1994. Iterative divergent/convergent approah to linear conjugated oligomers by successive doubling of the molecular length: A rapid route to a $128 \AA$ A-long potential molecular wire. Angew. Chem. Int. Ed. Engl. 33: 1360-1363.

30. Pearson, D.L., J.S. Schumm \& J.M. Tour. 1994. Iterative divergent/convergent approah to linear conjugated oligomers by a doubling of molecular length at each iteration. A rapid route to potential molecular wires. macromolecules 27: 23482350.

31. Pearson, D.L. \& J.M. Tour. 1997. Rapid syntheses of oligo(2,5-thiophene ethynylene)s with thioester termini: Potential molecular scale wires with alligator clips. J. Org. Chem. 62: 1376-1387.

32. Jones, L., II, J. S. Schumm \& J. M. Tour. 1997. Rapid solution and solid phase syntheses of oligo(1,4-phenylene ethynylene)s with thioester termini: Molecular scale wires with alligator clips. Derivatization of iterative reaction efficiences on a polymer support. J. Org. Chem. 62: 1388-1410.

33. YounG, J.K., J.C. NELSON \& J.S. MOORE. 1994. Synthesis of sequence specific phenylacetylene oligomers on an insoluble solid support. J. Am. Chem. Soc. 116: 10841-10842.

34. Bharathi, P. et al. 1995. Improvements in the synthesis of phenylacetylene monodendrons including a solid-phase convergent method. Macromolecules 28: 59555963.

35. Hodge, P. \& D.C. Sherrington, Eds. 1980. Polymer-Supported Reactions in Organic Synthesis. Wiley. New York, NY. In press.

36. Nelson, J.C., J.K. Young \& J.S. Moore. 1996. Solid-phase synthesis of phenylacetylene oligomers utilizing a novel 3-propyl-3-(benzyl-supported) Triazene Linkage. J. Org. Chem. 61: 8160-8168.

37. REED, M.A. et al. 1997. Conductance of a molecular junction. Science 278: 252 254.

38. ReINeRTh, W.A. \& J.M. TouR. 1997. Protecting groups for organoselenium compounds. J. Org. Chem. 63. 2397-2400.

39. ZHOU, C. et al. 1997. Nanoscale metal/self-assembled monolayer/metal heterostructures. Appl. Phys. Lett. 71: 611-613.

40. ZHOU, C. et al. 1997. Variation of a molecular alligator clip. Manuscript in preparation.

41. Seminario, J.M. \& J.M. Tour. Unpublished results. 